Check for updates

Cite this: RSC Adv., 2019, 9, 3504

Received 7th December 2018

Accepted 18th January 2019

DOI: $10.1039 / c 8 r a 10065 a$

rsc.li/rsc-advances

\section{Multifunctional amphiphilic ionic liquid pathway to create water-based magnetic fluids and magnetically-driven mesoporous silica $\nmid$}

\begin{abstract}
Jing Shen, (D) *a Wen $\mathrm{He}^{\mathrm{b}}$ and Tongwen Wang ${ }^{\mathrm{b}}$
Amphiphilic ionic liquids, 1-alkyl-3-methylimidazolium chloride $\left(\mathrm{C}_{n}\right.$ mimCl with $\left.n=10,12,14,16\right)$ were firstly used as modifiers to construct a self-assembly bilayer on the surface of iron oxide nanoparticles for generation of highly stable, water-based magnetic fluids. Subsequently, a magnet-driven mesoporous silica was synthesized by in situ self-assembly in the bilayer $\mathrm{C}_{n}$ mimCl-stabilized magnetic fluid using the $\mathrm{C}_{16} \mathrm{mimCl}$ as template and tetraethylorthosilicate (TEOS) as silicon source via a hydrothermal synthesis and following calcination procedure. A systematic study was carried out addressing the influence of the alkyl chain length of $\mathrm{C}_{n}$ mimCl in the primary and secondary layers on the stability of magnetic fluids. The characterization of TEM, XRD, VSM, electrophoresis experiments, TGA and DTA showed that stable water-based magnetic fluids can be synthesized based on the assembly of the well-defined bilayer$\mathrm{C}_{n}$ mimCl structure with long-chain $\mathrm{C}_{16}$ mimCl as secondary layer on the magnetite $\left(\mathrm{Fe}_{3} \mathrm{O}_{4}\right)$ nanoparticles. The results of small and wide-angle XRD, TEM, VSM, and $\mathrm{N}_{2}$ absorption experiments indicated that the nano-scale magnetic $\mathrm{Fe}_{3} \mathrm{O}_{4}$ particles were inlayed into hexagonal $\mathrm{p} 6 \mathrm{~mm}$ mesoporous silica (MCM-41 type) framework. Importantly, it was found that the obtained $\mathrm{Fe}_{3} \mathrm{O}_{4} / \mathrm{MCM}-41$ was an appropriate adsorbent for the adsorption of rhodamine $B$ and methylene blue from their aqueous solution. In addition, the adsorbent could be separated and reclaimed fleetly from the solution under external magnetic field.
\end{abstract}

\section{Introduction}

The use of ionic liquids has opened new avenues in the synthesis and fabrication of various new materials with advanced properties. ${ }^{1-3}$ Particularly, amphiphilic ionic liquids, composed of a charged hydrophilic imidazolium head group and a hydrophobic 'long tail' domain, can provide not only the amphiphilic properties of conventional surfactants, but also the specificity of typical short-chain room temperature ionic liquids such as low melting point, strong polarity and high resolvability in aqueous solution etc. Much work has been made to explore self-organized behaviour of amphiphilic ionic liquids in aqueous and other solvents. ${ }^{4-6}$ For example, Zheng and coworkers measured the micelle formation and the micelle aggregation numbers of amphiphilic ionic liquids in aqueous solution by surface tension, electrical conductivity and fluorescence measurement $e^{e t c}{ }^{7-9}$ They found that the surface activity of amphiphilic ionic liquids in aqueous solution is

${ }^{a}$ Department of Applied Chemistry, College of Vocational Education, Yunnan Normal University, Kunming 650092, China. E-mail: shenjingbox0225@hotmail.com

${ }^{b}$ College of Chemistry and Chemical Engineering, Yunnan Normal University, Kunming 650092, China

$\dagger$ Electronic supplementary information (ESI) available. See DOI: $10.1039 / \mathrm{c} 8 \mathrm{ra} 10065 \mathrm{a}$ slightly superior to that of the typical cationic surfactants. These self-organized advantages of amphiphilic ionic liquids have been applied to construct ordered mesoporous materials. For instance, ordered mesoporous silica with $2 \mathrm{D}$ hexagonal structure was synthesized by using amphiphilic ionic liquids, 1-alkyl3-methylimidazolium chloride $\left(\mathrm{C}_{n} \mathrm{mimCl}, n\right.$ is number of carbon atoms in alkyl chains), as templates. ${ }^{10}$ Zhou et al. reported a preparation of supermicroporous lamellar silica through 1-hexadecyl-3-methylimidazolium chloride $\left(\mathrm{C}_{16} \mathrm{mimCl}\right)$ as template. ${ }^{11}$ Our group has also contributed much effort to successfully synthesize mesoporous silica with high-quality cubic gyroid and 2D hexagonal mesoporous structures by using the $\mathrm{C}_{16} \mathrm{mimCl}$ as template. ${ }^{12}$ More recently, we have employed a $\mathrm{C}_{16}$ mimCl-assisted synthesis method to prepare polymer/Pd microspheres and a bimodal porous Pd-decorated silica. ${ }^{13}$ These research results confirmed that $\mathrm{C}_{n}$ mimCl possess a specific templating performance in the synthesis of ordered porous structural materials. However, their potential as modifying agent, especially for the formation of stable $\mathrm{C}_{n^{-}}$ mimCl-functionalized magnetic $\mathrm{Fe}_{3} \mathrm{O}_{4}$ nanoparticles (NPs) or their multifunctional combination for the fabrication of hybrid porous materials is less commonly known.

The aim of this work is twofold: firstly, it is intended to demonstrate that 1-alkyl-3-methylimidazolium chloride $\left(\mathrm{C}_{n^{-}}\right.$ $\mathrm{mimCl}$ ) can serve as a surface functional molecule to construct 
a bilayer of $\mathrm{C}_{n} \mathrm{mimCl}$ in the surface of magnetic $\mathrm{Fe}_{3} \mathrm{O}_{4}$ NPs, which can effectively produce a stable water-based magnetic fluid. Secondly, based on the $\mathrm{C}_{n}$ mimcl-based magnetic fluid, a more facile access to prepare magnetically-driven mesoporous silica with magnetic $\mathrm{Fe}_{3} \mathrm{O}_{4}$ NPs inlayed randomly into ordered hexagonal mesoporous silica framework by using $\mathrm{C}_{16} \mathrm{mimCl}$ as template and their adsorption properties is presented.

The $\mathrm{C}_{n}$ mimCl-bilayer structure onto $\mathrm{Fe}_{3} \mathrm{O}_{4} \mathrm{NPs}$ is similar to the surfactant-bilayer modified on the surface of iron oxide particles, which has been confirmed to form stable water-based magnetic fluid by effectively overcoming the agglomeration of $\mathrm{Fe}_{3} \mathrm{O}_{4}$ NPs. The combinations of various surfactants, such as fatty acid/fatty acid, ${ }^{\mathbf{1 4 , 1 5}}$ oleate sodium/PEG-4000, ${ }^{\mathbf{1 6}}$ oleic acid/ succinimide, ${ }^{17}$ and oleic acid/sodium dodecyl sulfate ${ }^{18}$ etc., have been used as modified bilayer to stabilize $\mathrm{Fe}_{3} \mathrm{O}_{4} \mathrm{NPs}$ in aqueous solution. In our design, the first research goal is to build a $\mathrm{C}_{n^{-}}$ mimCl-bilayer on the surface of $\mathrm{Fe}_{3} \mathrm{O}_{4}$ NPs. We expect that the $\mathrm{C}_{n}$ mimCl should exhibit the strong self-assembly ability on the surface of $\mathrm{Fe}_{3} \mathrm{O}_{4}$ NPs to form a high charge density and strong hydrophilic surface, which can form stable water-based magnetic fluid.

Recently, significant advances have been achieved in the synthesis of magnetic mesoporous silica materials because of their versatile application such as adsorption and separation, ${ }^{19}$ catalysis $^{20}$ and biological technology. ${ }^{21}$ In the synthesis of these magnetic mesoporous silica materials, the commonly used approach is based on the construction of a core-shell structure, in which magnetite particle services as inner core and a layer mesoporous silica acts as outer shell. ${ }^{21}$ This synthesis procedure is complicated, and it is difficult to accurately control the experimental conditions. It is also impossible to exclude the formation of some irregular pore structures. Thus, the exploitation of a convenient way of homogeneous incorporation of magnetic particles into the framework of mesoporous silica by optimized various methods is still a valuable research issue. Another objective of our research is to demonstrate the supramolecular templating function of $\mathrm{C}_{16} \mathrm{mimCl}$ for the preparation of highly ordered magnetic mesoporous silica in the prepared $\mathrm{Fe}_{3} \mathrm{O}_{4} / \mathrm{C}_{16} \mathrm{mimCl} / \mathrm{C}_{16}$ mimCl magnetic fluid. We expect that $\mathrm{C}_{n^{-}}$ mimCl can be manipulated in tandem as bilayer modifying agent and templating agent in one system, should provide important guidelines for the multifunctional applications of amphiphilic ionic liquids in the synthesis of various nanostructured materials.

\section{Experimental section}

Synthesis of $\mathrm{C}_{n} \operatorname{mimCl}(n=10,12,14,16)$

Amphiphilic ionic liquids, 1-alkyl-3-methylimidazolium chloride (abbreviated as $\mathrm{C}_{n} \mathrm{mimCl}, n$ is the number of carbon atoms in alkyl chains, $n=10,12,14,16$, respectively), were prepared according to a route reported in the literatures. ${ }^{13,22}$ All chemicals were purchased from Acrös and used as received. As a typical synthesis of $\mathrm{C}_{16} \mathrm{mimCl}$, an excess of 1-hexadecyl chloride $(33.41 \mathrm{~g}, 0.128 \mathrm{~mol})$ was mixed with 1-methylimidazole (10.26 g, $0.125 \mathrm{~mol}$ ). The mixture was put into a $250 \mathrm{~mL}$ flask, refluxed at $90{ }^{\circ} \mathrm{C}$ for $24 \mathrm{~h}$, and then cooled to room temperature. The product was further purified by recrystallization in tetrahydrofuran (THF). After being washed several times with THF, the white crystalline $\mathrm{C}_{16}$ mimCl powder was collected by filtration, and dried in air at room temperature. The preparation of the other $\mathrm{C}_{n}$ mimCl was achieved by repeating the above procedure with substitution of the corresponding 1-alkyl chloride for 1hexadecyl chloride, i.e., 1-decyl chloride for $\mathrm{C}_{10} \mathrm{mimCl}$, 1dodecyl chloride for $\mathrm{C}_{12} \mathrm{mimCl}$ and 1-tetradecyl chloride for $\mathrm{C}_{14}$ mimcl. The structures of the obtained $\mathrm{C}_{n}$ mimCl were identified by IR spectrum (see Fig. S1 $\dagger$ ).

\section{Synthesis of bilayer $\mathrm{C}_{\boldsymbol{n}}$ mimCl-stabilized magnetic fluids}

The bilayer $\mathrm{C}_{n}$ mimCl-stabilized magnetic fluids were synthesized by the chemical coprecipitation of $\mathrm{Fe}^{2+}$ and $\mathrm{Fe}^{3+}$ salts in $1: 2 \mathrm{M}$ ratio from a basic aqueous solution containing a small number of $\mathrm{C}_{n}$ mimCl, followed by the modifying process of the primary and secondary $\mathrm{C}_{n}$ mimCl, respectively. In a typical preparation of primary $\mathrm{C}_{10}$ mimCl-coated iron oxide particles, $0.86 \mathrm{~g}$ of $\mathrm{FeCl}_{2} \cdot 4 \mathrm{H}_{2} \mathrm{O}, 2.35 \mathrm{~g}$ of $\mathrm{FeCl}_{3} \cdot 6 \mathrm{H}_{2} \mathrm{O}$, and $0.1 \mathrm{~g}$ of $\mathrm{C}_{10}$ mimCl were dissolved in $40 \mathrm{~mL}$ of distilled water at $60{ }^{\circ} \mathrm{C}$ under mechanical paddle stirring and $\mathrm{N}_{2}$ purge. After $10 \mathrm{~min}$, the temperature was raised to $80{ }^{\circ} \mathrm{C}$, followed by $0.5 \mathrm{~mL}$ of $33 \%$ (w/w) $\mathrm{NH}_{4} \mathrm{OH}$ to adjust the $\mathrm{pH}$ value of the mixture to 9. After reaction for $10 \mathrm{~min}$, the $\mathrm{pH}$ value of the mixture was adjusted again to 13 by adding tetramethylammonium hydroxide dropwise, and then, $\mathrm{C}_{10} \mathrm{mimCl}$ was further added to the suspension in five $0.2 \mathrm{~g}$ amounts over $5 \mathrm{~min}$. The reaction was allowed to proceed for $30 \mathrm{~min}$ at $80{ }^{\circ} \mathrm{C}$ under constant stirring. Subsequently, the suspension was cooled slowly to room temperature and the precipitates were isolated from the solution by magnetic decantation. The precipitates were further purified with the magnetic decantation and redispersion in water and ethanol for three cycles. The resulted product was coded as $\mathrm{Fe}_{3} \mathrm{O}_{4} / \mathrm{C}_{10}$ mimCl. The other primary $\mathrm{C}_{n}$ mimCl-coated iron oxide particles were prepared by repeating above procedure with the corresponding $\mathrm{C}_{n}$ mimCl as primary layer modifier and coded as $\mathrm{Fe}_{3} \mathrm{O}_{4} / \mathrm{C}_{12} \mathrm{mimCl}, \mathrm{Fe}_{3} \mathrm{O}_{4} / \mathrm{C}_{14} \mathrm{mimCl}$ and $\mathrm{Fe}_{3} \mathrm{O}_{4} / \mathrm{C}_{16} \mathrm{mimCl}$, respectively. For comparison, bare $\mathrm{Fe}_{3} \mathrm{O}_{4}$ nanoparticles were prepared by above similar procedure without addition of any $\mathrm{C}_{n}$ mimcl.

To prepare stable water-based magnetic fluids, a series of secondary $\mathrm{C}_{n}$ mimCl with similar chemical structures to the primary $\mathrm{C}_{n}$ mimCl were coated on the primary $\mathrm{C}_{n}$ mimCl-coated iron oxide particles. In a typical preparation of $\mathrm{Fe}_{3} \mathrm{O}_{4} /$ $\mathrm{C}_{10} \mathrm{mimCl} / \mathrm{C}_{16} \mathrm{mimCl}$ magnetic fluid, $1 \mathrm{~mL}$ of $\mathrm{C}_{16} \mathrm{mimCl}$ solution $\left(40 \mathrm{~g} \mathrm{~L}^{-1}\right)$ was firstly added to $20 \mathrm{~mL}$ of distilled water. $0.5 \mathrm{~g}$ of $\mathrm{Fe}_{3} \mathrm{O}_{4} / \mathrm{C}_{10}$ mimCl obtained above was added to the solution under ultrasound at room temperature. After $10 \mathrm{~min}, \mathrm{C}_{16} \mathrm{mimCl}$ solution $\left(40 \mathrm{~g} \mathrm{~L}^{-1}\right)$ was further added dropwise to the suspension in $1 \mathrm{~mL}$ amounts over 5 min under vigorous mechanical paddle stirring until no visible settling and phase separation was observed when the solution was placed over a permanent magnet for a period of $5 \mathrm{~min}$. The sample was coded as $\mathrm{Fe}_{3} \mathrm{O}_{4} /$ $\mathrm{C}_{10}$ mimCl$/ \mathrm{C}_{16} \mathrm{mimCl}$. The preparations of the other bilayer structures were achieved by repeating the above procedure with corresponding bilayer combination of $\mathrm{C}_{n}$ mimCl as primary and 
secondary layers. In order to make subsequent various analyses, a small portion of dried magnetic nanoparticles can be separated from the magnetic fluids by a decantation procedure using a permanent magnet placed next to the bottle to provide a magnetic field of about $0.3 \mathrm{~T}$ over $3-4$ weeks.

\section{Synthesis of magnetic mesoporous silica}

The magnetic mesoporous silica was synthesized by a hydrothermal synthesis procedure in the $\mathrm{Fe}_{3} \mathrm{O}_{4} / \mathrm{C}_{16} \mathrm{mimCl} / \mathrm{C}_{16} \mathrm{mimCl}$ fluid using tetraethylorthosilicate (TEOS) as silicon source and $\mathrm{C}_{16} \mathrm{mimCl}$ as template. In a typical synthesis procedure, $\mathrm{C}_{16} \mathrm{mimCl}$ and $\mathrm{NaOH}$ were dissolved in the $\mathrm{Fe}_{3} \mathrm{O}_{4} / \mathrm{C}_{16} \mathrm{mimCl} /$ $\mathrm{C}_{16}$ mimCl fluid under mechanical paddle stirring. After homogenization of the mixture, TEOS was added dropwise at room temperature. The molar compositions of the starting $\begin{array}{llll}\text { mixtures were } & 1.0 & \text { TEOS : } x & \mathrm{Fe}_{3} \mathrm{O}_{4} / \mathrm{C}_{16} \mathrm{mimCl} / \mathrm{C}_{16}\end{array}$ mimCl : $0.1 \mathrm{C}_{16} \mathrm{mimCl}: 0.45 \mathrm{NaOH}: 140 \mathrm{H}_{2} \mathrm{O}(x=0.025,0.05$, 0.10 , respectively). The resulted mixtures were stirred at room temperature for $60 \mathrm{~min}$, and then transferred into a PTFE-lined steel autoclave and heated at $100{ }^{\circ} \mathrm{C}$ for 3 days. After hydrothermal treatment, the mixtures were filtered, washed with deionized water, dried under atmosphere at room temperature, and finally calcined at $550{ }^{\circ} \mathrm{C}$ for $5 \mathrm{~h}$ with a temperature ramp of $2{ }^{\circ} \mathrm{C} \min ^{-1}$ under static air conditions to remove the template. The final product, coded as $\mathrm{Fe}_{3} \mathrm{O}_{4} / \mathrm{MCM}-41$, was ground into powder for further characterization.

\section{Adsorption experiment}

The adsorption experiments were carried out by the adsorption of two dyes, rhodamine $\mathrm{B}(\mathrm{RhB})$ and methylene blue (MB), using the prepared $\mathrm{Fe}_{3} \mathrm{O}_{4} / \mathrm{MCM}-41$ as adsorbent at room temperature. For comparison, the adsorption experiments of two dyes on the pure mesoporous silica MCM- 41 (without $\mathrm{Fe}_{3} \mathrm{O}_{4} \mathrm{NPs}$ ) prepared using $\mathrm{C}_{16}$ mimCl template method which we reported earlier, ${ }^{\mathbf{1 2}}$ and on the bare $\mathrm{Fe}_{3} \mathrm{O}_{4}$ particles synthesized using the same chemical coprecipitation method without adding ionic liquid were also studied at room temperature. In a typical adsorption procedure, $\mathrm{Fe}_{3} \mathrm{O}_{4} / \mathrm{MCM}-41(0.1 \mathrm{~g})$ was added to the aqueous suspensions $(50 \mathrm{~mL})$ of the dye solutions with concentration of $x \mathrm{mg} \mathrm{L}^{-1}$ (where $x=10,20,50,100,200$, and $400 \mathrm{mg} \mathrm{L}^{-1}$, respectively) under mechanical stirring. At fixed intervals, the precipitates were isolated from the suspensions by magnetic decantation, and the absorbance of the solutions was measured by UV-vis spectrophotometer (SHIMADZU UV-1780) at 554 and $664 \mathrm{~nm}$ for RhB and MB solutions, respectively. The decolorization rates of the solutions were calculated according to eqn (1)

$$
D^{\mathrm{o}} \mathrm{=}=\left(A_{0}-A_{t}\right) \times 100 \% / A_{0}
$$

where $A_{0}$ and $A_{t}$ are absorbance values of the solution measured before and after the adsorption, respectively. The concentrations of the solutions were also determined by the standard working curves of two corresponding standard dye solutions. The adsorption capacity of $\mathrm{Fe}_{3} \mathrm{O}_{4} / \mathrm{MCM}-41, q_{t}\left(\mathrm{mg} \mathrm{g}^{-1}\right)$, at any time $t$ was determined using eqn (2)

$$
q_{t}=\left(C_{0}-C_{t}\right) V / m
$$

where $C_{0}$ and $C_{t}\left(\mathrm{mg} \mathrm{L}^{-1}\right)$ are the concentrations of the dye at initial time and time $t$, respectively. $V$ is the volume of solution (L), and $m$ is the amount of $\mathrm{Fe}_{3} \mathrm{O}_{4} / \mathrm{MCM}-41(\mathrm{~g})$. The experimental data were treated according to Freundlich isotherm equation eqn (3)

$$
q_{\mathrm{e}}=K_{\mathrm{F}} C_{\mathrm{e}}^{1 / n}
$$

where $q_{\mathrm{e}}$ is the equilibrium adsorption capacity $\left(\mathrm{mg} \mathrm{g}^{-1}\right) ; C_{\mathrm{e}}$ is the equilibrium concentration of dye solution $\left(\mathrm{mg} \mathrm{L}^{-1}\right) ; K_{\mathrm{F}}(\mathrm{mg}$ $\mathrm{g}^{-1}$ ) and $n$ are empirical constants which reflecting the adsorption capacity and heterogeneity factor, respectively. The linearized form of Freundlich isotherm can be expressed as eqn (4)

$$
\log q_{\mathrm{e}}=\log K_{\mathrm{F}}+(1 / n) \log C_{\mathrm{e}}
$$

Moreover, after the adsorption process, the precipitates were calcined at $550{ }^{\circ} \mathrm{C}$ for $5 \mathrm{~h}$ to remove the adsorbed dyes. The reusability of the recovered $\mathrm{Fe}_{3} \mathrm{O}_{4} / \mathrm{MCM}-41$ adsorbents was also studied by tracking the changes in UV-vis absorption spectrograph of the adsorption system under different cycles.

\section{Characterization}

Small- and wide-angle X-ray diffraction (XRD) patterns were measured on a TTR III powder X-ray diffractometer using $\mathrm{Cu} \mathrm{K} \alpha$ radiation (wavelength $0.154 \mathrm{~nm}$ ) at a rate of $0.05^{\circ} 2 \theta \mathrm{s}^{-1}$ and operated at $40 \mathrm{kV}$ and $30 \mathrm{~mA}$. Transmission electron microscopy (TEM) images were taken on a JEM-2100 electron microscope at an acceleration voltage of $200 \mathrm{kV}$. The specimens for TEM were prepared by dropping a small drop of the solutions onto a carbon-coated copper grid. Electrophoresis experiments were performed on a Nanjing Sangli DYL-3 electrophoresis apparatus with $15 \mathrm{~V}$ of external voltage. The magnetization of the samples was measured by a vibrating sample magnetometer (VSM 7407) at room temperature. Thermogravimetric analysis (TGA) and differential thermal analysis (DTA) were performed with a ZRY-1P thermal analysis system. Nitrogen sorption experiments were conducted using a Micromeritics Tristar 3000 automated gas adsorption analyzer. A TENSOR27 Fourier transform infrared (FT-IR) spectrometer was employed for recording IR spectra.

\section{Results and discussion}

Synthesis and characterization of $\mathrm{C}_{n}$ mimCl bilayer assembled onto $\mathrm{Fe}_{3} \mathrm{O}_{4}$ particles

As schematically depicted in Scheme 1, the strategy to prepare the hydrophilic magnetic $\mathrm{Fe}_{3} \mathrm{O}_{4} \mathrm{NPs}$ with ionic liquid $\mathrm{C}_{n} \mathrm{mimCl}$ bilayer as shell involves the first synthesis of $\mathrm{Fe}_{3} \mathrm{O}_{4}$ NPs, followed by coating magnetite NPs with $\mathrm{C}_{n} \operatorname{mimCl}(n=10,12,14$, 16) as inner layer and outer layers, respectively. The $\mathrm{Fe}_{3} \mathrm{O}_{4} \mathrm{NPs}$ were synthesized based on the well-established coprecipitation method using $\mathrm{Fe}^{2+}$ and $\mathrm{Fe}^{3+}$ salts from a basic aqueous solution. ${ }^{23}$ We found that during the precipitation process, the size 


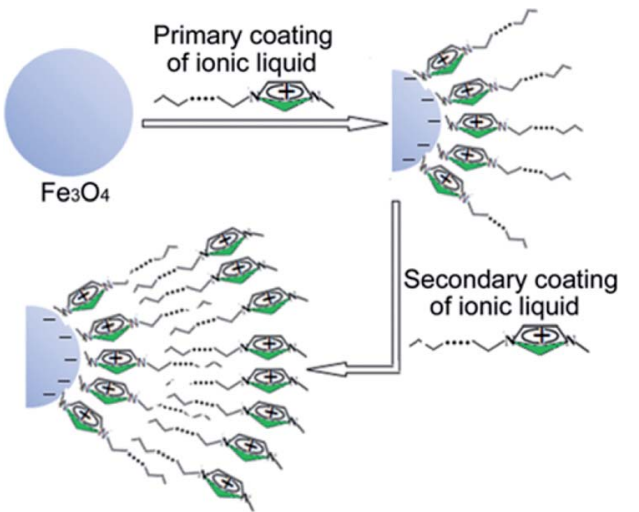

Scheme 1 Schematic diagram of the synthesis procedure of $\mathrm{Fe}_{3} \mathrm{O}_{4} /$ $\mathrm{C}_{n}$ mimCl/ $\mathrm{C}_{n}$ mimCl particle using $\mathrm{C}_{n}$ mimCl as bilayer modifier

of $\mathrm{Fe}_{3} \mathrm{O}_{4}$ NPs can be easily controlled with the initial presence of a small quantity of $\mathrm{C}_{n} \operatorname{mimCl}\left(2.5 \mathrm{~g} \mathrm{~L}^{-1}\right)$. Before coating primary $\mathrm{C}_{n}$ mimCl, the $\mathrm{pH}$ value of the suspension was adjusted to 13 by adding TMAOH, which led to negatively charged iron oxide particles. ${ }^{24}$ The precipitated $\mathrm{Fe}_{3} \mathrm{O}_{4}$ NPs were first coated with a primary layer of $\mathrm{C}_{n} \mathrm{mimCl}$ adsorbed to the iron oxide surfaces through an electrostatic attraction between the negatively charged $\mathrm{Fe}_{3} \mathrm{O}_{4}$ NPs and the positively charged imidazole heads of $\mathrm{C}_{n}$ mimCl. For example, the particles coated with monolayer $\mathrm{C}_{10} \mathrm{mimCl}$ were unstable and settled from the aqueous solution in a few minutes due to its hydrocarbon chains extending from the particle surface (Fig. S2a $\uparrow$ ). These particles were attracted by placing a magnet near the glass bottle, demonstrating that the particles possess magnetic properties (Fig. S2b $\dagger$ ). Compared with the frequently-used surfactants such as fatty acids that are sparingly soluble in water and their solubility was enhanced by the presence of acetone ${ }^{15} \mathrm{C}_{n} \mathrm{mimCl}$ is highly soluble in water because of its special hydrophilic imidazole head. The excellent solubility of $\mathrm{C}_{n} \mathrm{mimCl}$ in water provide the possibility of direct interactions between individual molecules of $\mathrm{C}_{n}$ mimCl and $\mathrm{Fe}_{3} \mathrm{O}_{4}$ particle, which is especially important for limiting the growth of $\mathrm{Fe}_{3} \mathrm{O}_{4}$ particle and preventing the agglomeration of the particles. Moreover, it was found that a drop by drop and slow adding way for the $\mathrm{C}_{n} \mathrm{mimCl}$ aqueous solution is especially important for preventing the micelle formation of $\mathrm{C}_{n} \mathrm{mimCl}$ in the dispersion. For example, the $\mathrm{cmc}$ of $\mathrm{C}_{16} \mathrm{mimCl}$ at $298 \mathrm{~K}$ was reported to be $1.21 \mathrm{mM},{ }^{25}$ and the concentration of one drop of the $\mathrm{C}_{16} \mathrm{mimCl}$ aqueous solution in the suspension is about $0.043 \mathrm{mM}$, which is well below the cmc of $\mathrm{C}_{16}$ mimCl. Hence, this adding way is favourable to coat the individual $\mathrm{C}_{16} \mathrm{mimCl}$ molecule onto $\mathrm{Fe}_{3} \mathrm{O}_{4}$ particles efficiently.

To prepare stable water-based magnetic fluids, $\mathrm{C}_{n}$ mimCl was secondly coated on the $\mathrm{Fe}_{3} \mathrm{O}_{4} / \mathrm{C}_{n}$ mimCl particles to form $\mathrm{Fe}_{3} \mathrm{O}_{4} /$ $\mathrm{C}_{n} \mathrm{mimCl} / \mathrm{C}_{n}$ mimCl particles. As shown in Fig. S2c, $\uparrow \mathrm{Fe}_{3} \mathrm{O}_{4} /$ $\mathrm{C}_{10} \mathrm{mimCl} / \mathrm{C}_{16} \mathrm{mimCl}$ colloidal suspension exhibited a remarkable stability as evidenced by a lack of observable precipitation over periods of more than 12 months. The zeta potential $(\zeta)$ of the $\mathrm{Fe}_{3} \mathrm{O}_{4} / \mathrm{C}_{10} \mathrm{mimCl} / \mathrm{C}_{16} \mathrm{mimCl}$ colloidal suspension was found to be approximately $+40 \mathrm{mV}$ measured by electrophoresis experiments. These results imply a hypothetical structure of the exposure of imidazole head groups of the secondary layer
$\mathrm{C}_{16}$ mimCl molecules toward the surrounding solution, and their hydrophobic long alkyl chain extending or inserting into the fence of alkyl chain of $\mathrm{Fe}_{3} \mathrm{O}_{4} / \mathrm{C}_{10} \mathrm{mimCl}$ by means of a hydrophobic interaction. The exposure of imidazole head groups in the surface of $\mathrm{Fe}_{3} \mathrm{O}_{4} / \mathrm{C}_{10}$ mimCl$/ \mathrm{C}_{16}$ mimCl provided high charge density and strong hydrophilic surfaces, resulting in the formation of stable and water-based magnetic fluid. We observed the formation of similar stable water-based magnetic fluids for $\mathrm{Fe}_{3} \mathrm{O}_{4} / \mathrm{C}_{12} \mathrm{mimCl} / \mathrm{C}_{16} \mathrm{mimCl}, \quad \mathrm{Fe}_{3} \mathrm{O}_{4} / \mathrm{C}_{14} \mathrm{mimCl} /$ $\mathrm{C}_{16} \mathrm{mimCl}$ and $\mathrm{Fe}_{3} \mathrm{O}_{4} / \mathrm{C}_{16} \mathrm{mimCl} / \mathrm{C}_{16} \mathrm{mimCl}$.

Fig. $1 \mathrm{~A}$ and $\mathrm{B}$ show the typical TEM images of ionic liquid bilayer-stabilized $\mathrm{Fe}_{3} \mathrm{O}_{4} / \mathrm{C}_{10} \mathrm{mimCl} / \mathrm{C}_{16} \mathrm{mimCl}$ and $\mathrm{Fe}_{3} \mathrm{O}_{4} /$ $\mathrm{C}_{16} \mathrm{mimCl} / \mathrm{C}_{16} \mathrm{mimCl}$ particles, respectively. It is clear that both magnetic oxides formed a well-dispersed quasi spherical morphology with clear boundary and mean diameter of approximately 10.5 and $10.1 \mathrm{~nm}$. Electron diffractions (the insets in Fig. 1A and B) measured from a large zone present a series of rings that can be indexed to the magnetite structure, which is consistent with the results obtained from wide-angle XRD patterns described in Fig. S3. $\dagger$

The magnetization curves of bare $\mathrm{Fe}_{3} \mathrm{O}_{4}, \mathrm{Fe}_{3} \mathrm{O}_{4} / \mathrm{C}_{10}$ mimCl, $\mathrm{Fe}_{3} \mathrm{O}_{4} / \mathrm{C}_{10} \mathrm{mimCl} / \mathrm{C}_{16}$ mimCl (Fig. 2A) and bare $\mathrm{Fe}_{3} \mathrm{O}_{4}, \mathrm{Fe}_{3} \mathrm{O}_{4} /$ $\mathrm{C}_{16} \mathrm{mimCl}, \mathrm{Fe}_{3} \mathrm{O}_{4} / \mathrm{C}_{16} \mathrm{mimCl} / \mathrm{C}_{16} \mathrm{mimCl}$ (Fig. 2B) displayed a typical magnetization " $\mathrm{S}$ " curve, where no reduced remanence and coercivity were observed, revealing the superparamagnetic nature of these magnetic particles. The saturation magnetization $\left(M_{\mathrm{s}}\right)$ of 74.2, 65.1 and $55.1 \mathrm{emu} \mathrm{g}^{-1}$ was determined for bare $\mathrm{Fe}_{3} \mathrm{O}_{4}, \quad \mathrm{Fe}_{3} \mathrm{O}_{4} / \mathrm{C}_{10} \mathrm{mimCl}$ and $\mathrm{Fe}_{3} \mathrm{O}_{4} / \mathrm{C}_{10} \mathrm{mimCl} / \mathrm{C}_{16} \mathrm{mimCl}$,

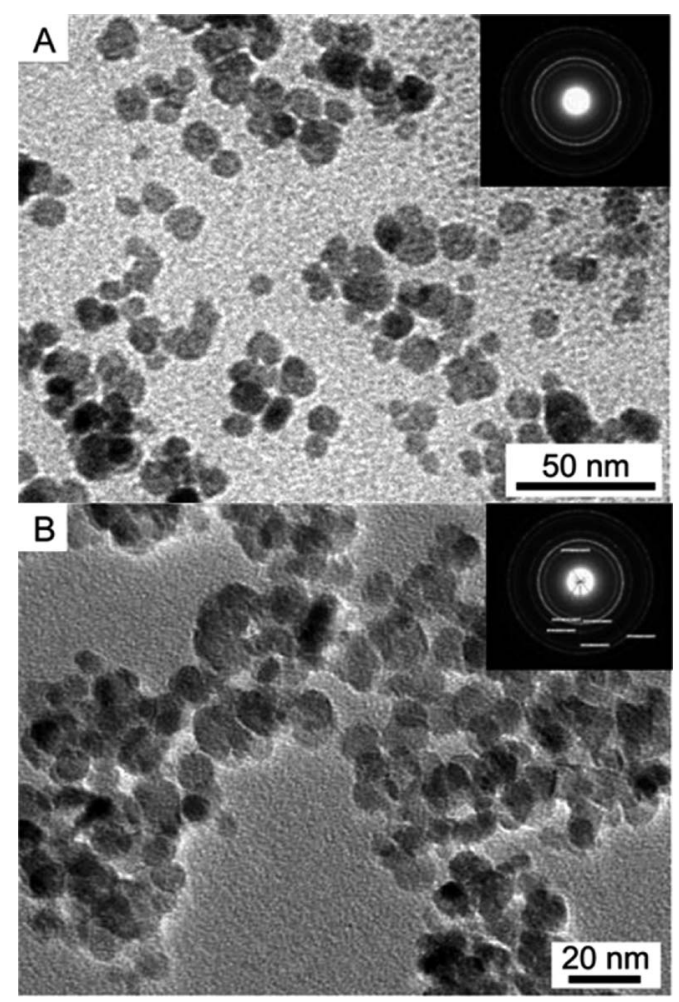

Fig. 1 TEM images of (A) ionic liquid bilayer-stabilized $\mathrm{Fe}_{3} \mathrm{O}_{4} /$ $\mathrm{C}_{10}$ mimCl/ $\mathrm{C}_{16} \mathrm{mimCl}$ and (B) $\mathrm{Fe}_{3} \mathrm{O}_{4} / \mathrm{C}_{16}$ mimCl/ $\mathrm{C}_{16} \mathrm{mimCl}$ particles. The insets show the corresponding electron diffraction images. 

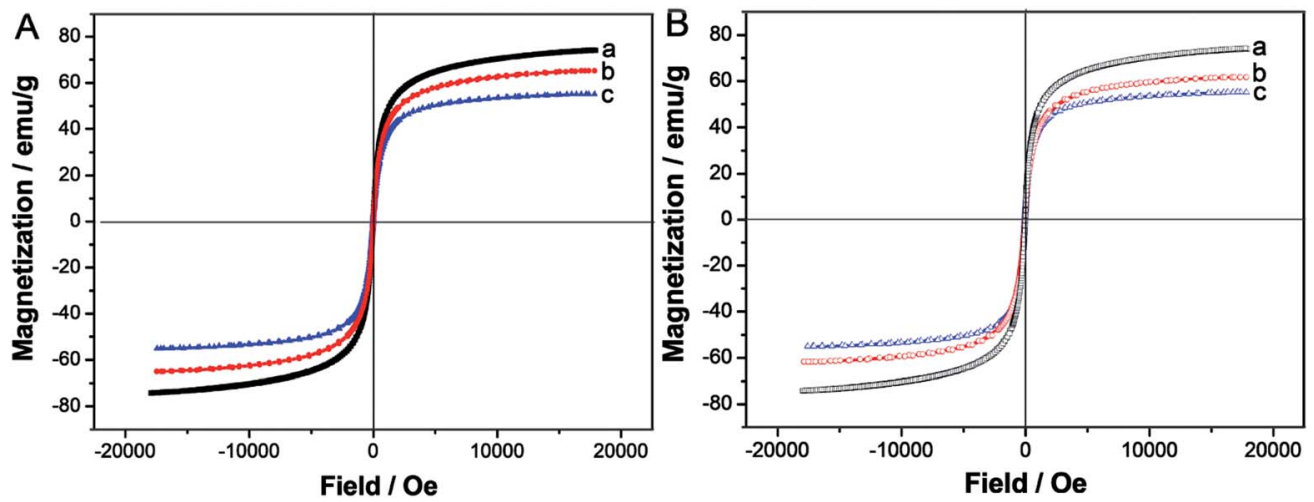

Fig. 2 (A) Magnetization curves of (a) bare $\mathrm{Fe}_{3} \mathrm{O}_{4}$, (b) $\mathrm{Fe}_{3} \mathrm{O}_{4} / \mathrm{C}_{10}$ mimCl, (c) $\mathrm{Fe}_{3} \mathrm{O}_{4} / \mathrm{C}_{10}$ mimCl/ $\mathrm{C}_{16}$ mimCl, and (B) magnetization curves of (a) bare $\mathrm{Fe}_{3} \mathrm{O}_{4}$, (b) $\mathrm{Fe}_{3} \mathrm{O}_{4} / \mathrm{C}_{16} \mathrm{mimCl}$, (c) $\mathrm{Fe}_{3} \mathrm{O}_{4} / \mathrm{C}_{16} \mathrm{mimCl} \mathrm{C}_{16} \mathrm{mimCl}$.

respectively. The similar results of the gradual reducing $M_{\mathrm{S}}$ from 74.2 to 61.8 and $55.0 \mathrm{emu} \mathrm{g}^{-1}$ can be observed for bare $\mathrm{Fe}_{3} \mathrm{O}_{4}$, $\mathrm{Fe}_{3} \mathrm{O}_{4} / \mathrm{C}_{16} \mathrm{mimCl}$ and $\mathrm{Fe}_{3} \mathrm{O}_{4} / \mathrm{C}_{16} \mathrm{mimCl} / \mathrm{C}_{16} \mathrm{mimCl}$ (see Fig. 2B). Obviously, the coating of primary $\mathrm{C}_{10} \mathrm{mimCl}$ (or $\mathrm{C}_{16} \mathrm{mimCl}$ ) layer onto $\mathrm{Fe}_{3} \mathrm{O}_{4}$ NPs led to a significant decrease in the values of $M_{\mathrm{s}}$, and the coating of secondary $\mathrm{C}_{16} \mathrm{mimCl}$ layer onto $\mathrm{Fe}_{3} \mathrm{O}_{4} /$ $\mathrm{C}_{10}$ mimCl (or $\mathrm{Fe}_{3} \mathrm{O}_{4} / \mathrm{C}_{16} \mathrm{mimCl}$ ) particles showed lower value of $M_{\mathrm{s}}$, i.e. $M_{\mathrm{s}}\left(\mathrm{Fe}_{3} \mathrm{O}_{4}\right)>M_{\mathrm{s}}\left(\mathrm{Fe}_{3} \mathrm{O}_{4} / \mathrm{C}_{10} \mathrm{mimCl}\right)>M_{\mathrm{s}}\left(\mathrm{Fe}_{3} \mathrm{O}_{4} / \mathrm{C}_{10} \mathrm{mimCl} /\right.$ $\mathrm{C}_{16} \mathrm{mimCl}$ ). These phenomena can be attributed to the detachment from dipole coupling due to the introduction of nonmagnetic species $\left(\mathrm{C}_{n} \mathrm{mimCl}\right)$ on the surface of $\mathrm{Fe}_{3} \mathrm{O}_{4}$ particles. ${ }^{26,27}$

To demonstrate the existence of $\mathrm{C}_{n}$ mimCl bilayer on the surface of iron oxide NPs, thermogravimetric analysis (TGA) and differential thermal analysis (DTA) were performed for the samples dried at room temperature. Fig. $3 \mathrm{~A}$ and B present the typical TGA and DTA curves of $\mathrm{Fe}_{3} \mathrm{O}_{4} / \mathrm{C}_{10}$ mimCl and $\mathrm{Fe}_{3} \mathrm{O}_{4} /$ $\mathrm{C}_{10} \mathrm{mimCl} / \mathrm{C}_{16} \mathrm{mimCl}$, respectively. As can be seen in Fig. $3 \mathrm{~A}$ (TGA curves), at the temperature below $150{ }^{\circ} \mathrm{C}$, both samples exhibited a small decrease step with a percentage weight loss of about $4.0 \%$ and $3.6 \%$ owing to the removal of adsorbed water in the samples. For the monolayer $\mathrm{C}_{10}$ mimCl-coated particles $\left(\mathrm{Fe}_{3} \mathrm{O}_{4} / \mathrm{C}_{10} \mathrm{mimCl}\right)$, a well-defined percentage weight loss of about $4.5 \%$ was detected over a temperature range of $170-$ $400{ }^{\circ} \mathrm{C}$. The weight loss should be assigned to desorption and subsequent combustion of the monolayer $\mathrm{C}_{10} \mathrm{mimCl}$ on the surface of particles. For the bilayer $\mathrm{C}_{10} \mathrm{mimCl} / \mathrm{C}_{16} \mathrm{mimCl}$-coated particles $\left(\mathrm{Fe}_{3} \mathrm{O}_{4} / \mathrm{C}_{10} \mathrm{mimCl} / \mathrm{C}_{16} \mathrm{mimCl}\right)$, a more significant percentage weight loss of about $12.7 \%$ was observed between 170 and $450{ }^{\circ} \mathrm{C}$. The difference of weight loss between $\mathrm{Fe}_{3} \mathrm{O}_{4} /$ $\mathrm{C}_{10} \mathrm{mimCl}$ and $\mathrm{Fe}_{3} \mathrm{O}_{4} / \mathrm{C}_{10} \mathrm{mimCl} / \mathrm{C}_{16} \mathrm{mimCl}$ can be attributed to the removal of the secondary $\mathrm{C}_{16} \mathrm{mimCl}$ shell layer. The DTA
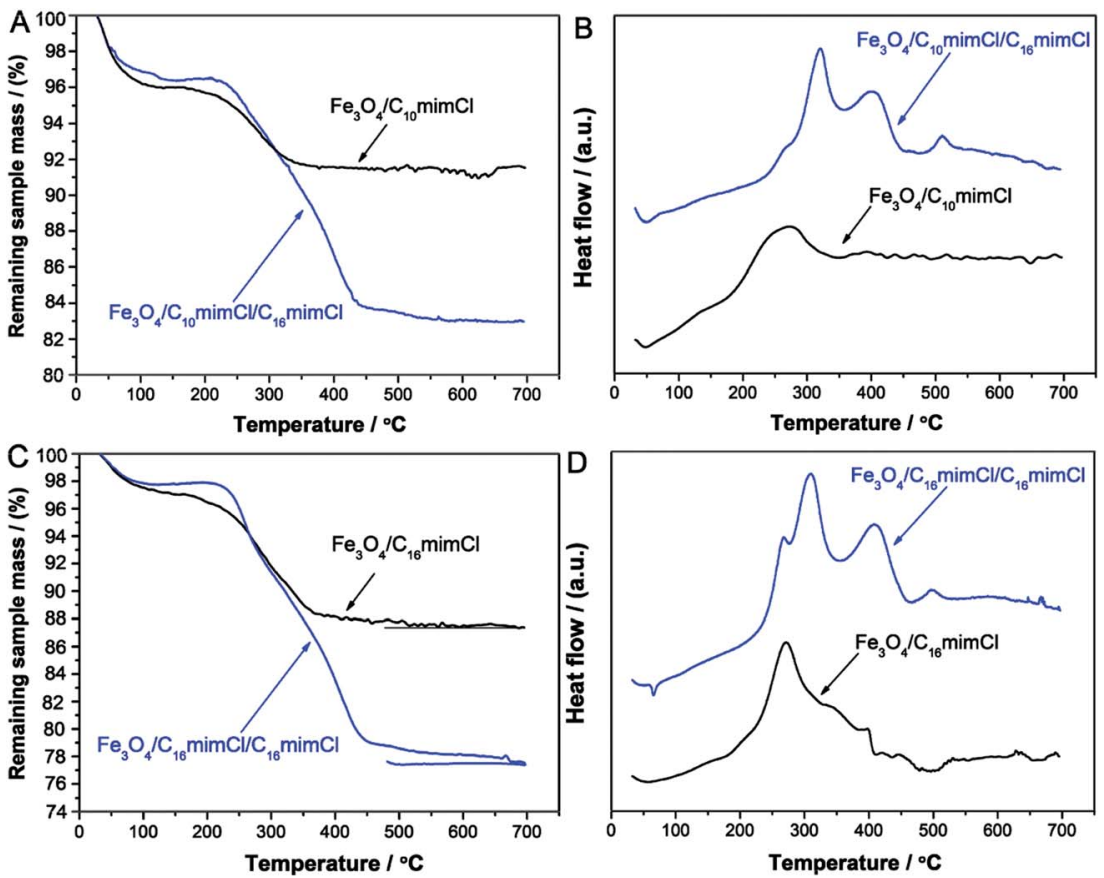

Fig. 3 (A) TGA and (B) DTA curves of $\mathrm{Fe}_{3} \mathrm{O}_{4} / \mathrm{C}_{10}$ mimCl and $\mathrm{Fe}_{3} \mathrm{O}_{4} / \mathrm{C}_{10}$ mimCl/C 16 mimCl. (C) TGA and (D) DTA curves of Fe $\mathrm{O}_{4} / \mathrm{C}_{16}$ mimCl and $\mathrm{Fe}_{3} \mathrm{O}_{4} / \mathrm{C}_{16} \mathrm{mimCl} / \mathrm{C}_{16} \mathrm{mimCl}$. 
Table 1 Coverage parameters of the monolayer and secondary layer $\mathrm{C}_{n}$ mimCl on $\mathrm{Fe}_{3} \mathrm{O}_{4}$ particle surface

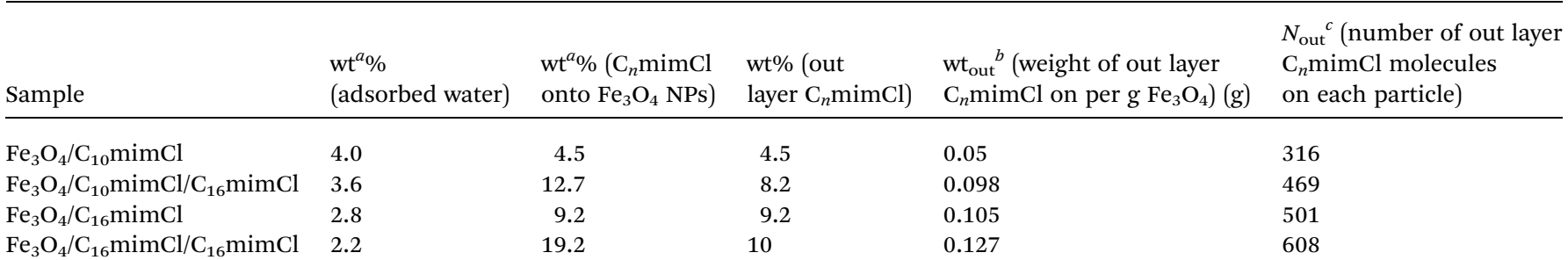

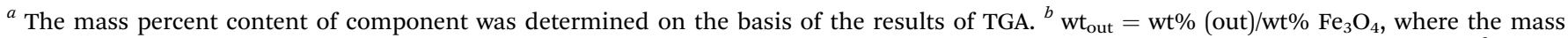
percent content of $\mathrm{Fe}_{3} \mathrm{O}_{4}$ was calculated by a formula that subtracts the mass percent of $\mathrm{C}_{n}$ mimCl and corresponding adsorbed water. ${ }^{c} N_{\text {out }}=$ $N_{1} / N_{2}$, where $N_{1}=$ wt $_{\text {out }} \times N_{\mathrm{A}} / M_{\text {out }}$ is the number of $\mathrm{C}_{n}$ mimCl molecules per $\mathrm{g} \mathrm{Fe} \mathrm{O}_{3}$, with $N_{\mathrm{A}}$ being the Avogadro constant and $M_{\text {out }}$ being the molar mass of out layer $\mathrm{C}_{n}$ mimCl, and $N_{2}=V_{1} / V_{2}$ is the number of $\mathrm{Fe}_{3} \mathrm{O}_{4}$ particles per $\mathrm{g} \mathrm{Fe}_{3} \mathrm{O}_{4}$, with $V_{1}=1 / \rho$ and $V_{2}=4 \pi R_{1}{ }^{3} / 3$ (or $V_{2}=4 \pi R_{2}{ }^{3}$ / 3) being the volume per $\mathrm{g} \mathrm{Fe}_{3} \mathrm{O}_{4}$ and the volume per particle, respectively, where $\rho$ is the density of $\mathrm{Fe}_{3} \mathrm{O}_{4}\left(5.18 \mathrm{~g} \mathrm{~cm}^{-3}\right)$ and $R_{1}$ is the radius of $\mathrm{Fe}_{3} \mathrm{O}_{4}$ particle, and $R_{2}=R_{1}+l$, with $l$ being the length of primary layer $\mathrm{C}_{n}$ mimCl.

curve (Fig. 3B) of $\mathrm{Fe}_{3} \mathrm{O}_{4} / \mathrm{C}_{10}$ mimCl exhibited one well-defined exothermic peak in the temperature range of $200-350{ }^{\circ} \mathrm{C}$, corresponding to decomposition of the monolayer $\mathrm{C}_{10} \mathrm{mimCl}$ on the surface of particles, while The DTA curve of $\mathrm{Fe}_{3} \mathrm{O}_{4} /$ $\mathrm{C}_{10} \mathrm{mimCl} / \mathrm{C}_{16} \mathrm{mimCl}$ (Fig. 3B) displayed two distinguishable exothermic peaks between 250 and $450{ }^{\circ} \mathrm{C}$, which might be due to the decomposition of the outer layer (the secondary layer) $\mathrm{C}_{16} \mathrm{mimCl}$ and the inner layer (the primary layer) $\mathrm{C}_{10} \mathrm{mimCl}$, respectively. A small exothermic peak at around $500-600{ }^{\circ} \mathrm{C}$ is possibly due to the structural transformation from magnetite to hematite when the temperature of magnetite NPs was exceeded $400{ }^{\circ} \mathrm{C}^{28}$ These observations can be easily explained in terms of the different molecular weights and boiling points of $\mathrm{C}_{10} \mathrm{mimCl}$ and $\mathrm{C}_{16} \mathrm{mimCl}$ molecules. Moreover, the two exothermic peaks taken place at different temperature can be assigned to the difference of interactions, such as electrostatic attraction between $\mathrm{C}_{10}$ mimCl and magnetite NPs, and hydrophobic interaction between the alkyl chain of $\mathrm{C}_{10} \mathrm{mimCl}$ and $\mathrm{C}_{16}$ mimCl. The similar results can be observed in Fig. $3 \mathrm{C}$ for $\mathrm{Fe}_{3} \mathrm{O}_{4} / \mathrm{C}_{16}$ mimCl (weight loss of about 9.2\%) and $\mathrm{Fe}_{3} \mathrm{O}_{4} /$ $\mathrm{C}_{16} \mathrm{mimCl} / \mathrm{C}_{16} \mathrm{mimCl}$ (weight loss of about $19.2 \%$ ), and Fig. 3D for $\mathrm{Fe}_{3} \mathrm{O}_{4} / \mathrm{C}_{16}$ mimCl with one exothermic peak and for $\mathrm{Fe}_{3} \mathrm{O}_{4} /$ $\mathrm{C}_{16} \mathrm{mimCl} / \mathrm{C}_{16} \mathrm{mimCl}$ with two exothermic peaks. These results revealed the existence of two different molecular layers coated on the iron oxide surface. Analogous to our results, Shen et al. observed the significant difference in the weight loss for monolayer and bilayer fatty acid surfactant-coated magnetite particles. ${ }^{15}$

We calculated further the coverage parameters of the monolayer $\mathrm{C}_{n}$ mimCl and the secondary layer $\mathrm{C}_{n} \mathrm{mimCl}$ on the $\mathrm{Fe}_{3} \mathrm{O}_{4}$ particle surface, respectively, in terms of the above TGA results. In these calculations, $\mathrm{Fe}_{3} \mathrm{O}_{4}$ particle was regard as spherical shape with mean diameter $D$ of $10 \mathrm{~nm}$ on the basis of the results of TEM (Fig. 1). The surface areas of the spheres were determined with diameters $D$ and $D+2 l$ for the monolayer and secondary coating of surfactants, respectively, ${ }^{15}$ with $l$ being the chain length of the primary surfactant. For example, the surfactant with chain length of $\mathrm{C}_{10}$ was estimated to be $c a$. $1.415 \mathrm{~nm}$ based on the method proposed by Shaw. ${ }^{29}$ Table 1 lists the coverage parameters of the monolayer and secondary layer $\mathrm{C}_{n}$ mimCl assembled with $\mathrm{C}_{10}$ mimCl and $\mathrm{C}_{16}$ mimCl on $\mathrm{Fe}_{3} \mathrm{O}_{4}$ particle surface. It is clear that the increase in the number of carbon atoms of the monolayer layer ionic liquid molecule from 10 to 16 increased the weight percent of the ionic liquid from 4.5 to 9.2, and the number of the ionic liquid molecules on each particle increased from 316 to 501. Meanwhile, the number of the $\mathrm{C}_{16} \mathrm{mimCl}$ molecules in secondary layer on each particle increased from 469 to 608 . These results demonstrated that the long-chain $\mathrm{C}_{16} \mathrm{mimCl}$ has stronger anchoring capacity on the iron oxide surface. The molecule number of 608 on each particle is close to that of the saturated fatty acids (e.g. myristic acid) adhered to iron oxide particle by a densely packed pattern. ${ }^{14} \mathrm{We}$ observed that the long-chain $\mathrm{C}_{16}$ mimCl exhibited a stronger assembly capability than that of the short-chain $\mathrm{C}_{10}$ mimCl for the secondary layer construction. The phenomenon can be explained on the basis of a competition between affinity of the imidazole head groups of $\mathrm{C}_{n} \mathrm{mimCl}$ for water and hydrophobic interaction between hydrocarbon tails of the primary and secondary $\mathrm{C}_{n}$ mimCl. It was known that the positively charged imidazole head groups possess intense hydrophilic properties. ${ }^{2}$ The shorter the hydrocarbon tail of $\mathrm{C}_{n} \mathrm{mimCl}$ is, the stronger the hydrophilic ability of $\mathrm{C}_{n}$ mimCl has. Hence, as the short-chain $\mathrm{C}_{10}$ mimCl was used as the secondary layer stabilizer, a highly affinity of water might make its molecules to have a tendency to remain in aqueous solution, resulting in a loose array of molecules on the particle surface. We found that the construction of $\mathrm{Fe}_{3} \mathrm{O}_{4} / \mathrm{C}_{10} \mathrm{mimCl} / \mathrm{C}_{10} \mathrm{mimCl}, \quad \mathrm{Fe}_{3} \mathrm{O}_{4} / \mathrm{C}_{12} \mathrm{mimCl} /$ $\mathrm{C}_{10} \mathrm{mimCl}, \mathrm{Fe}_{3} \mathrm{O}_{4} / \mathrm{C}_{14} \mathrm{mimCl} / \mathrm{C}_{10} \mathrm{mimCl}$ and $\mathrm{Fe}_{3} \mathrm{O}_{4} / \mathrm{C}_{16} \mathrm{mimCl} /$ $\mathrm{C}_{10}$ mimCl cannot produce stable magnetic fluids, and their particles settled from the aqueous solution in a few minutes. When the carbon atom number of the secondary layer $\mathrm{C}_{n} \mathrm{mimCl}$ increases to 16 , the hydrophobic interaction is evidently enhanced, leading to a significant increase in the assembly capability on the particle coated with the primary layer $\mathrm{C}_{n^{-}}$ mimCl. The bilayer $\mathrm{C}_{n}$ mimCl-coated $\mathrm{Fe}_{3} \mathrm{O}_{4} \mathrm{NPs}$, such as $\mathrm{Fe}_{3} \mathrm{O}_{4} /$ $\mathrm{C}_{16} \mathrm{mimCl} / \mathrm{C}_{16} \mathrm{mimCl}$, having close-packed imidazole headgroups exposing to the surrounding solution would provide a highly charge-density and strongly hydrophilic surface. The special structure surface can prevent the particles not only from aggregating but also from oxidizing through the electrostatic and steric repulsions between the particles, leading to the highly stable, water-based magnetic fluids. 


\section{Characterization of $\mathrm{C}_{16}$ mimCl-based magnetic mesoporous silica}

As shown in the Scheme 2, in the above synthesized $\mathrm{Fe}_{3} \mathrm{O}_{4} /$ $\mathrm{C}_{16} \mathrm{mim} / \mathrm{C}_{16} \mathrm{mim}$ magnetic fluid, a magnetic mesoporous silica was synthesized by a hydrothermal synthesis using $\mathrm{C}_{16} \mathrm{mimCl}$ as template and TEOS as silicon source. Fig. 4 shows smallangle XRD patterns of the calcined samples prepared from reaction mixtures with an initial molar ratio $n\left(\mathrm{Fe}_{3} \mathrm{O}_{4} / \mathrm{C}_{16} \mathrm{mimCl} /\right.$ $\left.\mathrm{C}_{16} \mathrm{mimCl}\right) / n$ (TEOS) of $0.025,0.05$ and 0.10 , respectively. It is seen that very similar diffraction patterns with three wellresolved characteristic peaks attributed to (100), (110) and (200) planes of $p 6 \mathrm{~mm}$ hexagonal structure (with reciprocal spacing, $1 / d_{h k l}$, ratios of $\left.31 / 2,2,71 / 2\right)$ were detected for two samples (Fig. 4a and b). These well-defined reflections peaks are in good agreement with the peaks of patterns from the siliceous MCM-41 prepared using quaternary ammonium ion surfactants, ${ }^{30}$ which suggests that the presence of magnetic particles with $n\left(\mathrm{Fe}_{3} \mathrm{O}_{4} / \mathrm{C}_{16} \mathrm{mimCl} / \mathrm{C}_{16} \mathrm{mimCl}\right) / n$ (TEOS) of 0.025 and 0.05 did not destroy the ordered array of mesoporous cannels. However, when the molar ratio was increased to 0.10 (Fig. 4c), only one well-resolved diffraction peak (100) in the $2 \theta$ range between 2 and 8 was detected, indicating that the ordered degree of mesopores is reduced. This is probably owing to the overfull addition of $\mathrm{Fe}_{3} \mathrm{O}_{4} / \mathrm{C}_{16} \mathrm{mimCl} / \mathrm{C}_{16} \mathrm{mimCl}$ particles, disrupting the charge density matching between the silicon species and $\mathrm{C}_{16} \mathrm{mimCl}$ liquid crystal phase, finally, leading to the partial breakdown of long-range ordered mesoporous silica structure. Generally, when guest species are loaded in the mesopores, the intensity of their XRD lines decreases. ${ }^{31}$

TEM images recorded along [100] (Fig. 5A) and [001] (Fig. 5B) directions for the calcined sample with $n\left(\mathrm{Fe}_{3} \mathrm{O}_{4} / \mathrm{C}_{16} \mathrm{mimCl} /\right.$ $\left.\mathrm{C}_{16} \mathrm{mimCl}\right) / n$ (TEOS) of 0.05 showed ordered hexagonal arrangement of mesopore channels as well as dark quasi spherical $\mathrm{Fe}_{3} \mathrm{O}_{4}$ NPs. These $\mathrm{Fe}_{3} \mathrm{O}_{4}$ NPs were enwrapped by the ordered mesoporous silica channels and distributed randomly throughout the mesoporous silica matrix. The TEM images provided a direct and conclusive evidence of the coexistence of $\mathrm{Fe}_{3} \mathrm{O}_{4}$ NPs and well-defined ordered mesoporous silica. Wideangle XRD pattern (Fig. S4A $\dagger$ ) of this sample measured at $2 \theta$ of $20-80^{\circ}$ presented some distinguishable weak Bragg peaks

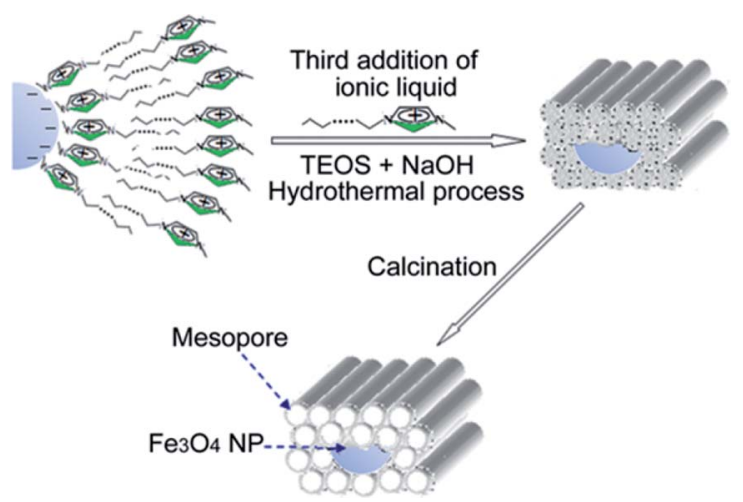

Scheme 2 Schematic diagram of prepared route of magnetic mesoporous silica using $\mathrm{C}_{16} \mathrm{mimCl}$ as template and TEOS as silicon source in the $\mathrm{Fe}_{3} \mathrm{O}_{4} / \mathrm{C}_{16}$ mimCl/ $\mathrm{C}_{16}$ mimCl magnetic fluid.

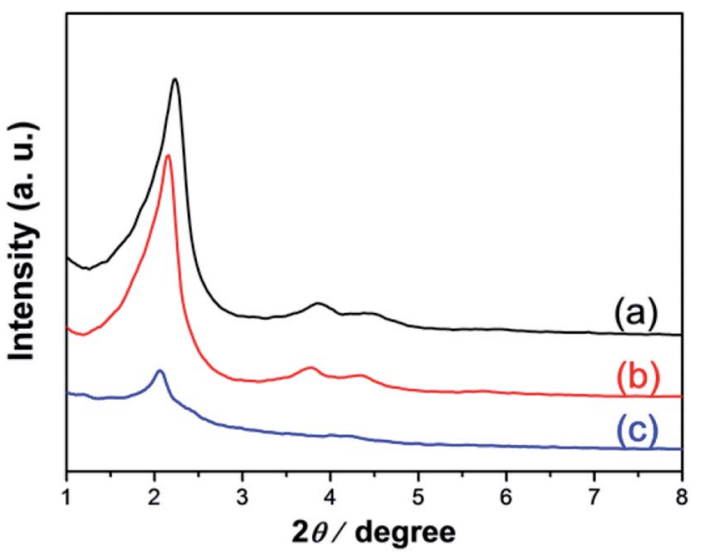

Fig. 4 Small-angle XRD patterns of calcined samples prepared using $\mathrm{C}_{16}$ mim $\mathrm{Cl}$ as template in the $\mathrm{Fe}_{3} \mathrm{O}_{4} / \mathrm{C}_{16}$ mimCl/ $\mathrm{C}_{16} \mathrm{mimCl}$ magnetic fluid with an initial molar ratio $n\left(\mathrm{Fe}_{3} \mathrm{O}_{4} / \mathrm{C}_{16} \mathrm{mimCl} / \mathrm{C}_{16} \mathrm{mimCl}\right) / n$ (TEOS) of (a) 0.025 , (b) 0.05 and (c) 0.10 .

that can be assigned to magnetite crystalline phase (JCPDS card no. 28-0491), which further confirms the existence of magnetite $\mathrm{Fe}_{3} \mathrm{O}_{4}$ NPs in the sample. A gradual protuberance at low angle can be attributed to amorphous silica of the pore walls. ${ }^{32}$ Magnetization curve of this sample shown in Fig. $\mathrm{S} 4 \mathrm{~B} \dagger$ exhibited also a typical magnetization "S" curve without obvious remanence and coercivity, revealing the superparamagnetic feature of the magnetic mesoporous silica.

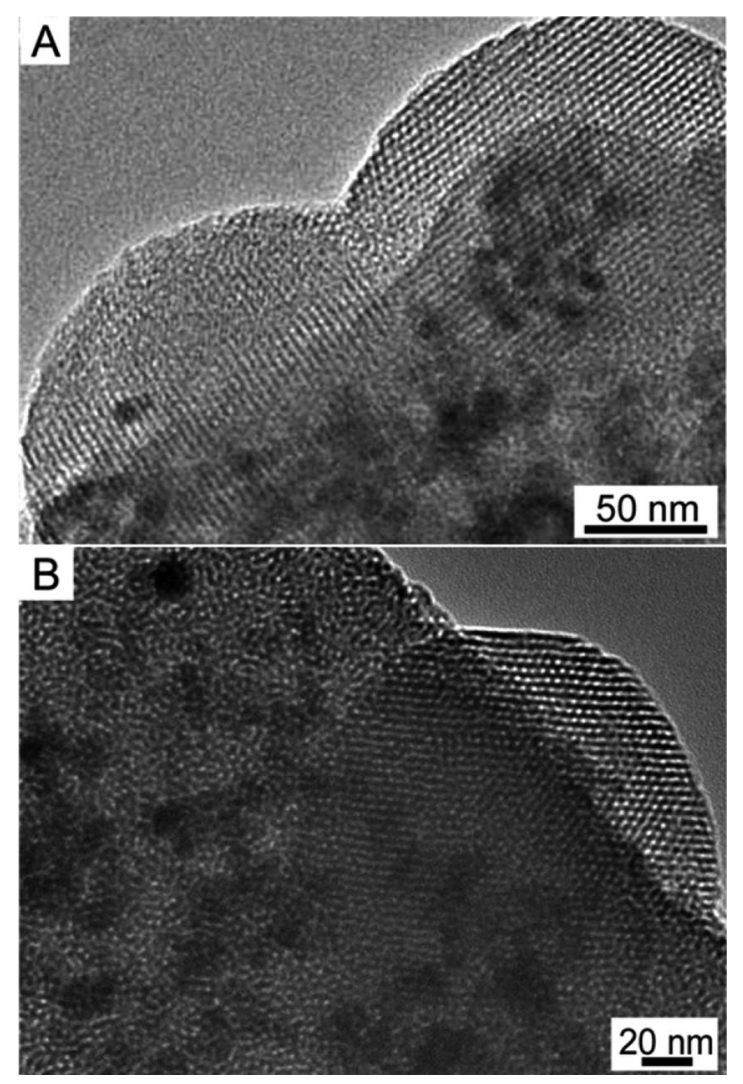

Fig. 5 TEM images recorded along the (A) [100] and (B) [001] directions of calcined sample prepared using $\mathrm{C}_{16}$ mimCl as template in the $\mathrm{Fe}_{3} \mathrm{O}_{4} / \mathrm{C}_{16} \mathrm{mimCl} / \mathrm{C}_{16} \mathrm{mimCl}$ magnetic fluid. 

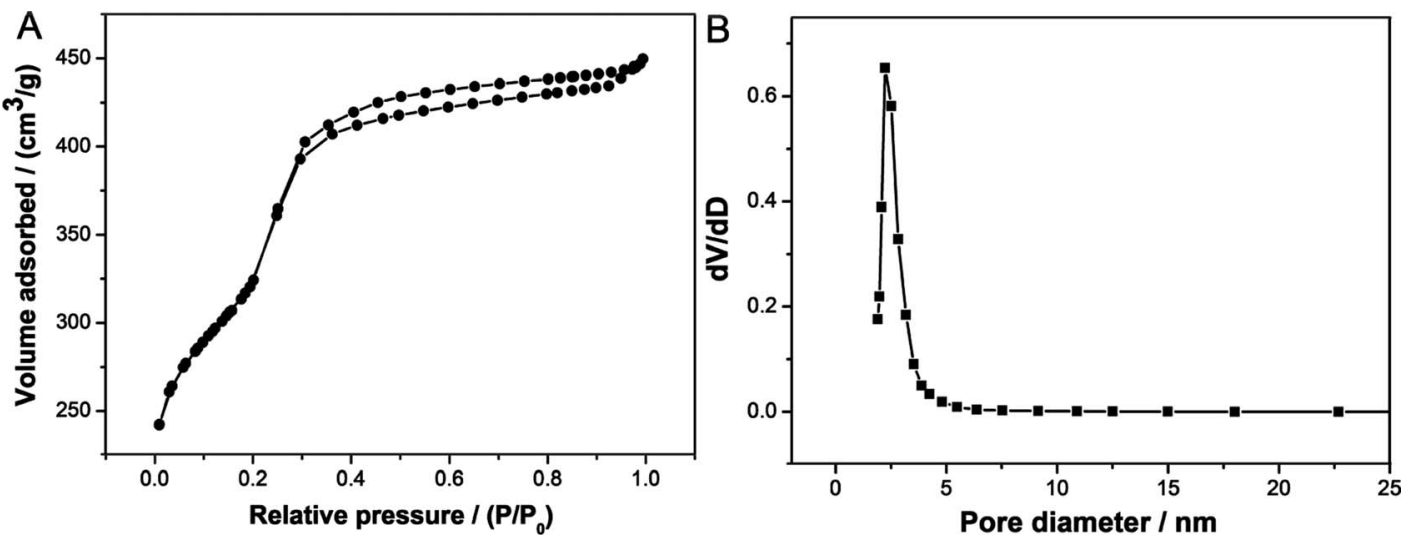

Fig. 6 (A) $\mathrm{N}_{2}$ adsorption-desorption isotherm of calcined sample prepared using $\mathrm{C}_{16} \mathrm{mimCl}$ as template in the $\mathrm{Fe}{ }_{3} \mathrm{O}_{4} / \mathrm{C}_{16} \mathrm{mimCl} / \mathrm{C}_{16}$ mimCl magnetic fluid, and (B) its $\mathrm{BJH}$ pore size distribution.

Nitrogen physisorption isotherm (Fig. 6A) of this sample displayed a type IV adsorption isotherm with an obvious hysteresis loop at a relatively high $p / p_{0}$ values according to IUPAC, ${ }^{33}$ indicating the presence of open pores. A steep increasing occurs at a relative pressure $0.30<p / p_{0}<0.43$, which is due to the filling of mesoporous walls by capillary condensation. In the previous publication, ${ }^{12}$ the hysteresis phenomenon was observed and attributable to the filling of a secondary pore structure, which resulted from grain boundaries, creating small cavities between adjacent ordered regions. In this sample, the $\mathrm{Fe}_{3} \mathrm{O}_{4}$ NPs can be regarded as grain, and its boundaries might create small cavities, therefore, resulting in the visible hysteresis loop. Pore size distribution calculated by BJH model (Fig. 6B) showed a narrow pore size distribution with the mean size of about $2.5 \mathrm{~nm}$, which is in agreement with the value from TEM images. The BET surface area and total pore volume are $915 \mathrm{~m}^{2} \mathrm{~g}^{-1}$ and $0.78 \mathrm{~cm}^{3} \mathrm{~g}^{-1}$, respectively.

\section{Adsorption of magnetic mesoporous silica}

To evaluate the adsorption properties of the prepared $\mathrm{Fe}_{3} \mathrm{O}_{4} /$ MCM-41, we employed rhodamine B (RhB) and methylene blue (MB) solutions as model systems at room temperature. Fig. 7 shows the time-dependent decolorization rates of two dye solutions measured at different initial concentrations after adsorption using the $\mathrm{Fe}_{3} \mathrm{O}_{4} / \mathrm{MCM}-41$ as adsorbent. It is clear that the decolorization rates of both solutions increased fast and reached a nearly plateau slope (about $90 \%$ ) within $10 \mathrm{~min}$ except for MB with initial concentration of $200 \mathrm{mg} \mathrm{L}^{-1}$. Fig. 8 shows the effect of contact time on the adsorption capacity of the $\mathrm{Fe}_{3} \mathrm{O}_{4} / \mathrm{MCM}-41$ in RhB (Fig. 8A) and $\mathrm{MB}$ (Fig. 8B) solutions with different initial concentrations of 20,50,100, 200 and $400 \mathrm{mg} \mathrm{L}^{-1}$, respectively. The two dyes were adsorbed rapidly in the initial $5 \mathrm{~min}$. After that, the adsorption capacity did not change with increasing of the contact time, indicating the adsorption equilibrium was approached. For adsorption of $\mathrm{RhB}$, the adsorption capacity of the $\mathrm{Fe}_{3} \mathrm{O}_{4} / \mathrm{MCM}-41$ in $\mathrm{RhB}$ solutions with different initial concentrations of 20, 50, 100, 200 and $400 \mathrm{mg} \mathrm{L}^{-1}$ at equilibrium (30 $\mathrm{min}$ ) can reach 9.74, 24.4, 48.8, 98.0 and $196 \mathrm{mg} \mathrm{g}^{-1}$, respectively (Fig. 8A). Compared with the maximum adsorption capacity $\left(q_{\mathrm{m}}=196 \mathrm{mg} \mathrm{g}^{-1}\right)$ of the $\mathrm{Fe}_{3} \mathrm{O}_{4} / \mathrm{MCM}-41$, the pure mesoporous silica MCM-41 had higher adsorption capacity $\left(q_{\mathrm{m}}=393 \mathrm{mg} \mathrm{g}^{-1}\right)$, which is exactly two times that of the $\mathrm{Fe}_{3} \mathrm{O}_{4} / \mathrm{MCM}-41$. The reason is probably that the specific surface area $\left(1200 \mathrm{~m}^{2} \mathrm{~g}^{-1}\right)^{12}$ of the pure MCM- 41 is
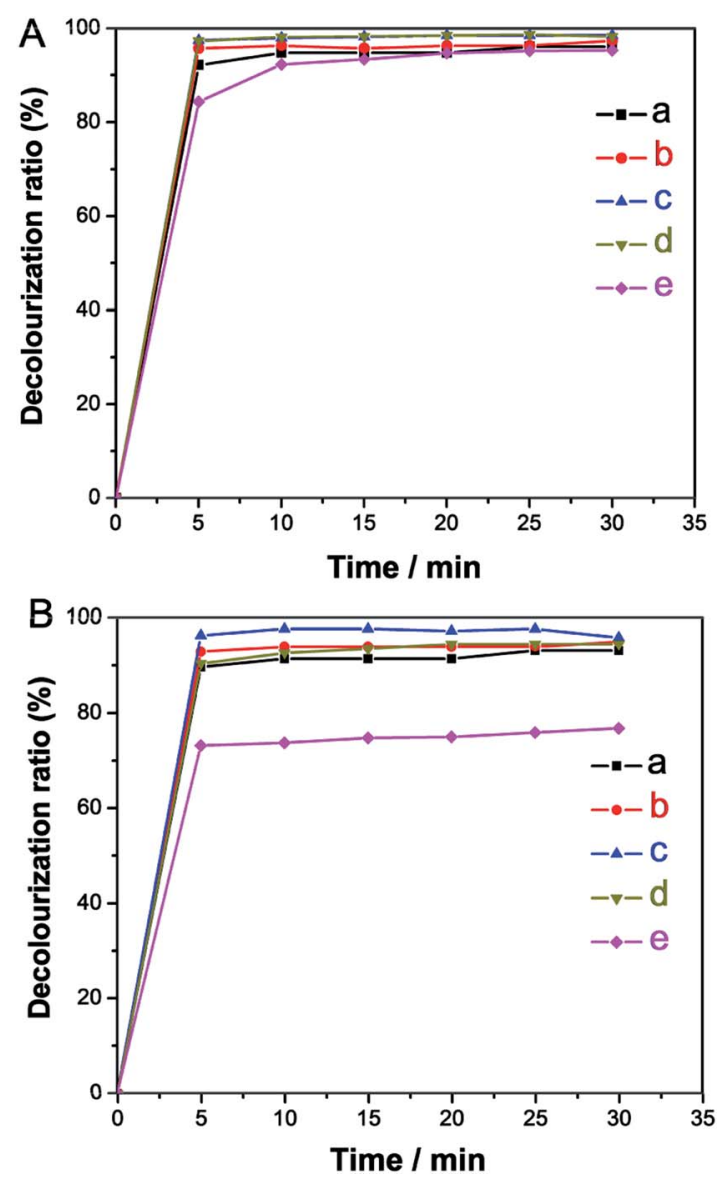

Fig. 7 Decolorization rates of (A) rhodamine $B$ and (B) methylene blue solutions after adsorption using $\mathrm{Fe}_{3} \mathrm{O}_{4} / \mathrm{MCM}-41$ as adsorbent and magnetic separation at different time interval. The initial concentrations of solutions for rhodamine B: (a) 20, (b) 50, (c) 100, (d) 200 (e) $400 \mathrm{mg} \mathrm{L}^{-1}$, and for methylene blue: (a) 10 , (b) 20, (c) 50, (d) 100 (e) $200 \mathrm{mg} \mathrm{L}^{-1}$. 

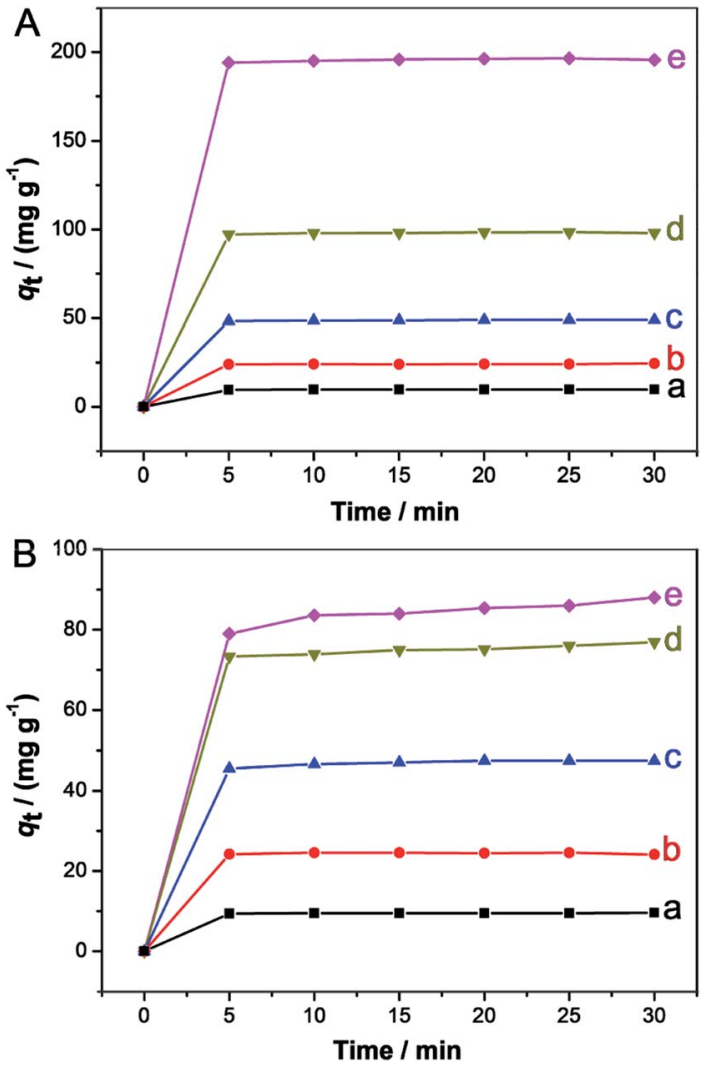

Fig. 8 Adsorbed capacity of $\mathrm{Fe}_{3} \mathrm{O}_{4} / \mathrm{MCM}-41$ as a function of contact time for (A) RhB and (B) MB at different initial concentrations of (a) 20, (b) 50 , (c) 100 , (d) 200 (e) $400 \mathrm{mg} \mathrm{L}^{-1}$ at room temperature.

higher than that of the $\mathrm{Fe}_{3} \mathrm{O}_{4} / \mathrm{MCM}-41\left(915 \mathrm{~m}^{2} \mathrm{~g}^{-1}\right)$. However, the pure mesoporous silica MCM-41 can only be recovered from the solution by centrifugation, while the $\mathrm{Fe}_{3} \mathrm{O}_{4} / \mathrm{MCM}-41$ can be easily recovered by magnetic separation technology. The $q_{\mathrm{m}}$ of the bare $\mathrm{Fe}_{3} \mathrm{O}_{4}$ particles prepared using the same chemical coprecipitation method without adding ionic liquid was found to be $7.5 \mathrm{mg} \mathrm{g}^{-1}$, which is lower than that of the $\mathrm{Fe}_{3} \mathrm{O}_{4} / \mathrm{MCM}-41$. This may be attributed to the fact that the $\mathrm{Fe}_{3} \mathrm{O}_{4} / \mathrm{MCM}-41$ has adequate mesoporous channels.

Similarly, for adsorption of $\mathrm{MB}$, the adsorption capacity of the $\mathrm{Fe}_{3} \mathrm{O}_{4} / \mathrm{MCM}-41$ in $\mathrm{MB}$ solutions with different initial concentrations of $20,50,100,200$ and $400 \mathrm{mg} \mathrm{L}^{-1}$ at equilibrium (30 $\mathrm{min}$ ) was found to be 9.62, 24.1, 47.5, 76.9 and $88.0 \mathrm{mg}$ $\mathrm{g}^{-1}$, respectively (Fig. 8B). It is clear that when the initial concentration of dyes is less than $100 \mathrm{mg} \mathrm{L}^{-1}$, the adsorption capacity of the $\mathrm{Fe}_{3} \mathrm{O}_{4} / \mathrm{MCM}-41$ for both dye solutions on is close. However, when the initial concentration of dyes was increased from 200 to $400 \mathrm{mg} \mathrm{L}^{-1}$, the adsorption capacity of the $\mathrm{Fe}_{3} \mathrm{O}_{4}$ / MCM-41 increased from 98.0 to $196 \mathrm{mg} \mathrm{g}^{-1}$ for RhB solution, while for MB solution, the value increased slowly from 76.9 to $88.0 \mathrm{mg} \mathrm{g}^{-1}$. It can be seen that for both dye solutions with high concentration $\left(\geq 200 \mathrm{mg} \mathrm{g}^{-1}\right.$ ), the $\mathrm{Fe}_{3} \mathrm{O}_{4} / \mathrm{MCM}-41$ is more beneficial for adsorption of $\mathrm{RhB}$ than $\mathrm{MB}$. This phenomenon may be attributed to the fact the $\mathrm{Fe}_{3} \mathrm{O}_{4} / \mathrm{MCM}-41$ is more likely to adsorb $\mathrm{RhB}$ with larger molecular structure because of the special mesoporous structure of the $\mathrm{Fe}_{3} \mathrm{O}_{4} / \mathrm{MCM}-41$. It was also
Table 2 Recycling of the adsorbents

\begin{tabular}{lll}
\hline Dye & Cycle & Decolorization rates (\%) \\
\hline \multirow{2}{*}{ Rhodamine B } & 1 & 95 \\
& 2 & 88.5 \\
& 3 & 82.1 \\
Methylene blue & 4 & 72.2 \\
& 1 & 93 \\
& 2 & 87.6 \\
& 3 & 80.5 \\
& 4 & 73.7 \\
\hline
\end{tabular}

found that for the adsorption of $\mathrm{MB}$, the $q_{\mathrm{m}}$ of $97.5,88.0$ and $5.50 \mathrm{mg} \mathrm{g}^{-1}$ was determined for pure MCM-41, $\mathrm{Fe}_{3} \mathrm{O}_{4} / \mathrm{MCM}-41$ and bare $\mathrm{Fe}_{3} \mathrm{O}_{4}$ particles, respectively, which showed a similar trend with that of the adsorption of RhB.

The well-defined linear relations (see Fig. S5†), fitted by Freundlich isotherm equation were observed for RhB solution in the range of $20-400 \mathrm{mg} \mathrm{L}^{-1}$ and for $\mathrm{MB}$ solution in the range of $10-100 \mathrm{mg} \mathrm{L}^{-1}$ adsorbed on the $\mathrm{Fe}_{3} \mathrm{O}_{4} / \mathrm{MCM}-41$. The obtained values of $K_{\mathrm{F}}$ and $1 / n$ were found to be $20 \mathrm{mg} \mathrm{g}^{-1}$ and 1.074 for $\mathrm{RhB}$, and $11 \mathrm{mg} \mathrm{g}^{-1}$ and 0.957 for $\mathrm{MB}$, respectively. Obviously, the adsorption capacity of the $\mathrm{Fe}_{3} \mathrm{O}_{4} / \mathrm{MCM}-41$ to $\mathrm{RhB}$ is higher than that of MB.

These results of adsorption analyses imply that the $\mathrm{Fe}_{3} \mathrm{O}_{4}$ / MCM-41 has luxuriant mesoporous channels and high surface area, and can be potentially used as an adsorbent in liquidphase processes. It is well-known that silicon-based MCM-41 mesopores have abundant and negatively charged surface silicon hydroxyl groups, which can adsorb the positively charged cationic dye, such as $\mathrm{RhB}$ or $\mathrm{MB}$, by means of an electrostatic adsorption.

The magnetic separability of the dye-adsorbed $\mathrm{Fe}_{3} \mathrm{O}_{4} / \mathrm{MCM}$ 41 adsorbents was tested by placing a conventional laboratory magnet near the small beaker containing the mixtures of two dye solutions (100 $\mathrm{mg} \mathrm{L}^{-1}$ ) and the adsorbent powder. As shown in Fig. S6, $\dagger$ after a certain time interval, the powder was attracted by the magnet (on the right), and the colour of the powder changed from grey to orange-red (Fig. S6A $\dagger$ ) and grey to blue (Fig. S6B $\dagger$ ) for RhB-adsorbed and $\mathrm{MB}$-adsorbed $\mathrm{Fe}_{3} \mathrm{O}_{4} /$ MCM-41, respectively. The clear solution could be decanted off or removed by pipette. These simple experiments confirmed that the $\mathrm{Fe}_{3} \mathrm{O}_{4} / \mathrm{MCM}-41$ is magnetic and can be used as a magnetic adsorbent to remove dyes in liquid-phase.

The regeneration of the $\mathrm{Fe}_{3} \mathrm{O}_{4} / \mathrm{MCM}-41$ was conducted by calcination of the dye-adsorbed $\mathrm{Fe}_{3} \mathrm{O}_{4} / \mathrm{MCM}-41$ powder at $550{ }^{\circ} \mathrm{C}$ for $5 \mathrm{~h}$ to remove the dyes from the pores. The reclaimed powder can be used as an adsorbent again. Table 2 shows the decolorization rates of $\mathrm{RhB}$ and $\mathrm{MB}$ solutions $\left(100 \mathrm{mg} \mathrm{L}^{-1}\right)$ after four cycles. After the three cycles, the decolorization rates of both dye solutions can be maintained at more than $80 \%$. However, after the fourth cycle, the decolorization rates were reduced to about $70 \%$. The phenomenon may be caused by partial collapse of the mesoporous structure of the adsorbent after several cycles. 


\section{Conclusions}

In summary, amphiphilic 1-alkyl-3-methylimidazolium chloride $\left(\mathrm{C}_{n} \mathrm{mimCl}\right)$ can serve as a surface functional molecule to construct bilayer $\mathrm{C}_{n}$ mimCl structure in the surface of magnetic $\mathrm{Fe}_{3} \mathrm{O}_{4}$ nanoparticles. It was found that $\mathrm{C}_{n} \operatorname{mimCl}(n=10,12,14$, 16) can be used to assemble the primer layer, and long chain $\mathrm{C}_{16}$ mimCl is favourable for the construction of the secondary layer. The bilayer $\mathrm{C}_{n}$ mimCl-based $\mathrm{Fe}_{3} \mathrm{O}_{4}$ particles provide a highly charge-density and strongly hydrophilic surface, leading to the highly stable, water-based magnetic fluids. Moreover, in the $\mathrm{C}_{n}$ mimCl-based magnetic fluid, a magnetically-driven mesoporous silica with magnetic $\mathrm{Fe}_{3} \mathrm{O}_{4}$ NPs inlayed randomly into ordered hexagonal mesoporous silica (MCM-41 type) framework can be prepared by using $\mathrm{C}_{16} \mathrm{mimCl}$ as template. Furthermore, the obtained $\mathrm{Fe}_{3} \mathrm{O}_{4} / \mathrm{MCM}-41$ with high surface area of up to $915 \mathrm{~m}^{2} \mathrm{~g}^{-1}$ exhibited enhanced decolorization rates (about 95\%) within $5 \mathrm{~min}$ for the rhodamine $\mathrm{B}$ and methylene blue from their aqueous solutions $\left(100 \mathrm{mg} \mathrm{\textrm {L } ^ { - 1 }}\right)$. This work may provide a new approach for the development of multifunctional amphiphilic ionic liquid in the practical nanomaterial applications.

\section{Conflicts of interest}

There are no conflicts to declare.

\section{Acknowledgements}

We gratefully acknowledge the financial support from National Natural Science Foundation of China (21564018, 21063017 and 21363029), and Yunnan Provincial Science and Technology Department (2015FD014).

\section{References}

1 K. R. Seddon, Nat. Mater., 2003, 22, 363-365.

2 M. Antonietti, D. Kuang, B. Smarsly and Y. Zhou, Angew. Chem., Int. Ed., 2004, 43, 4988-4992.

3 B. Xin and J. Hao, Chem. Soc. Rev., 2014, 43, 7171-7187.

4 M. Blesic, M. H. Marques, N. V. Plechkova, K. R. Seddon, L. P. N. Rebelo and A. Lopes, Green Chem., 2007, 9, 481-490.

5 J. Łuczak, J. Hupka, J. Thöming and C. Jungnickel, Colloids Surf., A, 2008, 329, 125-133.

6 Y. Pei, L. Hao, J. Ru, Y. Zhao, H. Wang, G. Bai and J. Wang, J. Mol. Liq., 2018, 254, 130-136.

7 B. Dong, N. Li, L. Zheng, L. Yu and T. Inoue, Langmuir, 2007, 23, 4178-4182.

8 T. Inoue, H. Ebina, B. Dong and L. Zheng, J. Colloid Interface Sci., 2007, 314, 236-241.
9 B. Dong, X. Zhao, L. Zheng, J. Zhang, N. Li and T. Inoue, Colloids Surf., A, 2008, 317, 666-672.

10 C. J. Adams, A. E. Bradley and K. R. Seddon, Aust. J. Chem., 2001, 54, 679-681.

11 Y. Zhou and M. Antonietti, Adv. Mater., 2003, 15, 1452-1455.

12 T. Wang, H. Kaper, M. Antonietti and B. Smarsly, Langmuir, 2007, 23, 1489-1495.

13 T. Wang, T. Fu, Y. Meng, J. Shen and T. Wang, RSC Adv., 2018, 8, 25141-25149.

14 A. Wooding, M. Kilner and D. B. Lambrick, J. Colloid Interface Sci., 1991, 144, 236-242.

15 L. F. Shen, P. E. Laibinis and T. A. Hatton, Langmuir, 1999, 15, 447-453.

16 R. Y. Hong, S. Z. Zhang, Y. P. Han, H. Z. Li, J. Ding and Y. Zheng, Powder Technol., 2006, 170, 1-11.

17 B. Bateer, Y. Qu, X. Meng, C. Tian, S. Du, R. Wang, K. Pan and H. Fu, J. Magn. Magn. Mater., 2013, 332, 151-156.

18 M. Soleymani and M. Edrissi, J. Dispersion Sci. Technol., 2016, 37, 693-698.

19 S. Hou, X. Li, H. Wang, M. Wang, Y. Zhang, Y. Chi and Z. Zhao, RSC Adv., 2017, 7, 51993-52000.

20 Q. Y. Li, K. R. Ma, Z. J. Ma, Q. Wei, J. G. Liu, S. P. Cui and Z. R. Nie, Microporous Mesoporous Mater., 2018, 265, 18-25.

21 N. Z. Knezevic, E. Ruiz-Hernandez, W. E. Hennink and M. Vallet-Regi, RSC Adv., 2013, 3, 9584-9593.

22 Y. Zhou and M. Antonietti, Chem. Mater., 2004, 16, 544-550. 23 R. Massart and V. Cabuil, J. Chem. Phys., 1987, 84, 967-973. 24 E. Dubois, J. Chevalet and R. Massart, J. Mol. Liq., 1999, 83, 243-254.

25 C. Jungnickel, J. Łuczak, J. Ranke, J. F. Fernández, A. Müller and J. Thfiming, Colloids Surf., A, 2008, 316, 278-284.

26 J. Wang, Q. Chen, C. Zeng and B. Hou, Adv. Mater., 2004, 16, 137-140.

27 D. Wang, C. Cao, S. Xue and H. Zhu, J. Cryst. Growth, 2005, 277, 238-245.

28 N. Pinna, S. Grancharov, P. Beato, P. Bonville, M. Antonietti and M. Niederberger, Chem. Mater., 2005, 17, 3044-3049.

29 D. J. Shaw, Introduction to Colloid and Surface Chemistry, Butterworths, London, 3rd edn, 1980, p. 90.

30 C. T. Kresge, M. E. Lconowiez, W. J. Roth, J. C. Vartuli and J. S. Beck, Nature, 1992, 359, 710-712.

31 C. K. Krishnan, T. Hayashi and M. Ogura, Adv. Mater., 2008, 20, 2131-2136.

32 M. Thommes, R. Köhn and M. Fröba, J. Phys. Chem. B, 2000, 104, 7932-7943.

33 K. S. W. Sing, D. H. Everett, R. A. W. Haul, L. Moscou, R. A. Pierotti, J. Rouquérol and T. Siemieniewska, Pure Appl. Chem., 1985, 57, 603-619. 